\title{
Algunos psicofármacos disminuyeron la reincidencia de episodios de violencia en presos excarcelados
}

\author{
Some psychotropic drugs decreased the recidivism of episodes of violence in released prisoners
}

\section{Comentado de:}

Chang Z, y col. JAMA. 2016 Nov 1;316(17):1798-1807. doi: 10.1001/jama.2016.15380 ${ }^{1}$.

\section{Objetivos}

Investigar los principales psicofármacos prescriptos en los presidiarios y examinar su asociación con el riesgo de reincidencia de episodios de violencia luego de la excarcelación.

\section{Diseño, lugar y tiempo}

Estudio de cohorte prospectiva, realizado en Suecia, que incluyó a todos los prisioneros liberados de la Swedish Prison and Probation Service (SPPS). El estudio se inició en julio de 2005 y finalizó en diciembre de 2010, obteniéndose la información de los registros poblacionales. Se compararon las tasas de reincidencia en episodios de violencia en períodos con medicación psicofarmacológica y periodos sin medicación a través del análisis en cada individuo y entre individuos.

\section{Participantes}

Se incluyeron 22.275 prisioneros liberados de la SPPS, edad promedio de 38 años, el 91,9\% de sexo masculino.

\section{Medición de resultados principales}

El resultado principal fue cualquier hecho de crimen violento (homicidio, asalto, robo, incendio provocado, violaciones, delitos de índole sexual, intimidación y amenazas) cometido luego de la excarcelación.

\section{Resultados principales}

Se halló una reducción substancial en las tasas de reincidencia en episodios de violencia en los individuos que recibieron antipsicóticos, psicoestimulantes y drogas para los trastornos adictivos (ver Tabla 1).

Esta diferencia se mantuvo en los subgrupos de prisioneros liberados con diagnóstico de esquizofrenia, trastorno bipolar, trastorno por déficit de atención e hiperactividad y trastornos adictivos.

Tabla 1. Asociaciones entre psicofármacos y reincidencia en episodios de violencia en los mismos individuos.

\begin{tabular}{|c|c|c|c|c|c|}
\hline \multirow[t]{2}{*}{ Madirarión } & \multicolumn{2}{|c|}{ Periodos con medicación } & \multicolumn{2}{|c|}{ Periodos sin medicación } & \multirow{2}{*}{ Haztard ratin IIS a5 o, } \\
\hline & $\mathrm{N}$ & Episodios & $\mathrm{N}$ & Episodios & \\
\hline Antipsicóticos & 2.085 & 100 & 2.767 & 1.044 & $0,58(0,39$ a 0,88$)$ \\
\hline Antidepresivos & 5.660 & 224 & 7.421 & 2.038 & $1,09(0,83$ a 1,43$)$ \\
\hline Psicoestimulantes & 1.202 & 94 & 1.352 & 513 & $0,62(0,40$ a 0,98$)$ \\
\hline $\begin{array}{l}\text { Drogas para los trastor- } \\
\text { nos adictivos }\end{array}$ & 2.077 & 46 & 3.055 & 1.103 & $0,48(0,23$ a 0,97$)$ \\
\hline Antiepilépticos & 2.235 & 152 & 2.736 & 800 & $1,14(0,79$ a 1,65$)$ \\
\hline
\end{tabular}

\section{Conclusiones}

Las tasas de reincidencia en episodios de violencia fueron menores en los períodos en los cuales los individuos recibieron medicación antipsicótica, psicoestimulantes y drogas para los trastornos adictivos, comparado con períodos en los cuales no recibieron estas medicaciones.
Fuente de financiamiento: Becas del Wellcome Trust, Swedish Research Council for Health, Working Life and Welfare, Swedish Initiative for Research on Microdata in the Social and Medical Sciences, European Union Seventh Framework Programme and the National Institute of Mental Health.

\section{Comentario}

La evidencia actual respecto del efecto de los psicofármacos sobre la conducta violenta es escasa. Los datos existentes en la literatura se basan en estudios descriptivos que utilizan una metodología poco sistematizada, las muestras suelen ser pequeñas y los resultados poco precisos. Algunas de las principales dificultades son el reclutamiento de los pacientes agresivos o violentos, la obtención de su consentimiento para participar de los estudios clínicos y el seguimiento de los mismos ${ }^{2}$.

Si bien las revisiones sistemáticas en relación a los efectos de los antipsicóticos mostraron disminución de las reinternaciones en pacientes con esquizofrenia, trastorno bipolar, depresión resistente y trastorno límite de la personalidad, la evidencia sobre 
los efectos de la farmacoterapia en otras áreas como el comportamiento violento es débil ${ }^{2}$.

Los psicofármacos más frecuentemente usados en los individuos excarcelados del estudio fueron los antidepresivos y antiepilépticos, sin embargo no se encontraron efectos de estos fármacos en la reducción de los episodios de violencia, lo cual concuerda con otros estudios realizados ${ }^{2-4}$.

El trabajo no investiga la naturaleza de los episodios de violencia en cada individuo. La categorización de los mismos permitiría el desarrollo de estrategias adecuadas y, en el caso de patología psiquiátrica, la selección de la farmacoterapia para cada individuo.

Dado que el estudio analizado es observacional no es posible establecer una fuerte relación causal entre los psicofármacos y la conducta violenta. Por otra parte el estudio fue realizado en un país con características sociodemográficas diferentes a nuestra población, por lo tanto no se pueden extrapolar los resultados de manera directa.

\section{Conclusiones del comentador}

El estudio abre la posibilidad de considerar a algunos psicofármacos como estrategia útil para evitar la reincidencia de episodios de violencia en los presos liberados. Sin embargo, habría que conocer más las características de los individuos seleccionados, los factores psicosociales involucrados, así como también los psicofármacos utilizados, para poder arribar a conclusiones.

Jose Luis Faccioli, Cecilia Jorgelina De Simone [ Servicio de Psiquiatría, Hospital Italiano de Buenos Aires, Buenos Aires, Argentina jose.faccioli@ hospitaltialiano.org.ar, cecilia.desimone@hospitalitaliano.org.ar ]

Faccioli JL, De Simone CJ. Algunos psicofármacos disminuyeron la reincidencia de episodios de violencia en presos excarcelados Evid Actual Pract Ambul. 2019;22(1):e001070. Comentado de: Chang Z. y col. Association Between Prescription of Major Psychotropic Medications and Violent Reoffending After Prison Release. JAMA.2016; 316 (17):1798-1807. PMID:27802545

\section{Referencias}

1. Chang Z, Lichtenstein P, Langström N, Larsson H, Fazel S. Association between prescription of major psychotropic medications and violent reoffending after prison release. JAMA - Journal of the American Medical Association. 2016;Available from: 10.1001/jama.2016.15380.

2. Fazel S, Zetterqvist J, Larsson $\mathrm{H}$, Långström N, Lichtenstein P. Antipsychotics, mood stabilisers, and risk of violent crime. Lancet. 2014;384(9949):1206-1214. Available from: 10.1016/S0140-6736(14)60379-2;https://www.thelancet.com/journals/lancet/article/PIIS0140-6736(14) 60379-2/fulltext.

3. Huband N, Ferriter N, Nathan R, Jones H, et al. Antiepileptics for aggression and associated impulsivity. Cochrane Database Syst Rev. 2010;17(2):CD003499. Available from: 10.1002/14651858.CD003499.pub3.

4. Bouvy PF, Liem M. Antidepressants and lethal violence in the Netherlands 1994-2008. Psychopharmacology. 2012;222(3):499-506. Available from: 10.1007/s00213-012-2668-2;https://link.springer.com/article/10.1007/s00213-012-2668-2. 